\title{
REGULARITY AND NUMERICAL SOLUTION FOR THE EXACT CONTROL
}

Mauro Antonio Rincon ${ }^{1}$

\begin{abstract}
In this paper we study a Numerical Method based on Finite Difference to the Boundary Exact Controllability problem for the Wave Equation. We consider the onedimensional problem. A regularity result is proven in order to allow us to use the Finite Difference Method. Numerical results are shown and compared to the exact solution of some particular problems, computed by the Fourier Serie.
\end{abstract}

Keywords: Wave Equation, Exact Controllability Problem, Finite Difference and Fourier Serie.

\section{INTRODUCTION}

The main goal of this paper is to develop a Numerical Method, using the technique of Finite difference in order to get approximate solution to the Exact Controllability Problem on the Boundary of the Wave Equation, considering the one-dimensional case. The numerical method described here consist in a combination of the implicit finite difference schemes, applied to the Wave Equation twice. The theory of the Exact Controllability has been introduced by J. L. Lions [2] and studied by several authors, as in [6], [7], [10]. In [3], a Numerical Method was developed for the same problem with $\Omega$ being a bounded domain of $I R^{n}$. In this case the solution is not continuous at the boundary, since it is devided in two parts. In the one-dimensional case we prove that the solution is continuous and has sufficient regularity for the application of the Finite Difference method. In order to compare the results, we study a problem of exact control in which the boundary values as well as the exact solution obtained by the Fourier Method 'are known. At the end of this paper, we show the graphs and tables for comparison.

1 Instituto de Matemática - UFRJ; Caixa Postal 68530 - CEP 21945 - 970; Río de Janeiro - Brasil. E-mail: rincon@lci.ufrj.br. 


\section{FORMULATION OF THE EXACT CONTROL PROBLEM}

We consider the following problem:

Determine $y=y(x, t)$, that satisfies

$$
\mid \begin{array}{ll}
y_{t}-y_{x x}=0 & \text { in }] 0, L[\times] 0, T[ \\
y(x, 0)=y_{0}(x) ; \quad y^{\prime}(x, 0)=y_{1}(x) & \text { in }] 0, L[ \\
y(0, t)=v(t) ; \quad y(L, t)=w(t) & \text { in }] 0, T[
\end{array}
$$

where $v$ and $w$ are functions which define the transversal motion on the boundary $x=0$ and $x=L$ respectively and are not known from the begining. Our problem is to determine the conditions for $v$ and $w$ such that at the end of a time $T>0$ (least possible) the equilibrium condition is true:

$$
y(x, T)=y^{\prime}(x, T)=0, \quad \forall x \in \Omega .
$$

\section{Description of the Hilbert Uniqueness Method - (HUM):}

Consider $\left\{\theta^{0}, \theta^{1}\right\} \in F$ and $\theta(x, t)$ as the solution of the probłem:

$$
\mid \begin{array}{ll}
\theta_{t t}-\theta_{x x}=0 & \text { in }] 0, L[\times] 0, T[ \\
\theta(x, 0)=\theta^{0}(x) ; \theta^{\prime}(x, 0)=\theta^{1}(x) & \text { in }] 0, L[ \\
\theta(0, t)=\theta(L, t)=0 & \text { in }] 0, T[.
\end{array}
$$

We define the retrograde problem, given by:

$$
\mid \begin{array}{ll}
\psi_{t t}-\psi_{x x}=0 & \text { in }] 0, L[\times] 0, T[ \\
\psi(x, T)=\psi_{t}(x, T)=0 & \text { in }] 0, L[ \\
\psi(0, t)=-\theta_{x}(0, t), & \psi(L, t)=\theta_{x}(L, t) .
\end{array}
$$

Consider the operator $\Lambda$, defined by:

$$
\Lambda\left\{\theta^{0}, \theta^{1}\right\}=\left\{\psi^{\prime}(x, 0),-\psi(x, 0)\right\}
$$

where $\left\{\theta^{0}, \theta^{1}\right\} \in F$. The fundamental result, to solve problem, is given by:

Lemma 2.1.- Let $F=H_{0}^{1}(0, L) \times L^{2}(0, L)$. For $T>\frac{L}{2}$ the operator $\Lambda$ is an isomorphism between the spaces $F$ and $F^{\prime}=H^{-1}(0, L) \times L^{2}(0, L)$.

For a proof of the Lemma, see [2] and [10]. From Lemma, it follows that it is sufficient to have $\left\{\psi^{\prime}(\dot{x}, 0),-\psi(x, 0)\right\} \in F^{\prime}$. Taking the controls $v(t)=-\theta_{x}(0, t)$ and $w(t)=\theta_{x}(L, t)$, then $y(x, t)$ and $\psi(x, t)$ are weak solutions for the same no homogeneous problem. As the problem has unique solution, follows that $y(x, t)=\psi(x, t)$. Therefore we conclude that $y(x, t)$ satisfies: $y(x, T)=y^{\prime}(x, T)=0$. We have then the theorem: 
Theorem 2.1.- For all pair of the initial data $\left\{y^{0}, y^{1}\right\} \in L^{2}(0, L) \times H^{-1}(0, L)$ and $T>\frac{L}{2}$ there are controls $v$ and $w$ in the $L^{2}(0, L)$ such that the solution $y=y(x, t)$ of the problem (1), satisfies the condition (2). Therefore $y=y(x, t)$ has a regularity:

$$
y \in C^{0}\left(0, T ; L^{2}(0, L)\right) \cap C^{1}\left(0, T ; H^{-1}(0, L)\right) .
$$

The Hilbert Uniqueness Method and the thecniques multiplicatily allow us to obtain more regularity on the solution, as shown on the following theorem:

Theorem 2.2.- For all pair of the initial values $\left\{y^{0}, y^{1}\right\} \in H_{0}^{1}(0, L) \times L^{2}(0, L)$ and $T>\frac{L}{2}$, there are controls $v$ and $w$ in $H_{0}^{1}(0, T)$ such that the solution $y=y(x, t)$ of the problem (1), satisfies the equilibrium condition (2).

Proof: We consider $F^{\prime}=H_{0}^{1}(0, L) \times L^{2}(0, L)$ and $\left\{\theta^{0}, \theta^{1}\right\} \in L^{2}(0, L) \times H^{-1}(0, L)$. We define

$$
\varphi=\int_{0}^{1} \theta(x, t) d t+\chi
$$

where $\chi \in H_{0}^{1}(0, L)$ and satisfies $\chi_{x x}=\theta^{1} \in H^{-1}(0, L)$. Integrating the equation (3) on $t$, we have

$$
\theta_{t}(x, t)-\theta_{t}(x, 0)-\int_{0}^{t} \theta_{x x}(x, t) d t=0 .
$$

From (7) we have that

$$
\varphi_{t t}(x, t)=\theta_{t}(x, t)
$$

and

$$
\varphi_{x x}(x, t)=\int_{0}^{1} \theta_{x x}(x, t) d t+\chi_{x x} .
$$

Substituting (9) and (10) in (8), we obtain

$$
\varphi_{t t}-\varphi_{x x}+\chi_{x x}-\theta^{1}=0
$$

Then the function $\varphi(x, t)$ satisfies;

$$
\mid \begin{aligned}
& \varphi_{t t}-\varphi_{x x}=0 \\
& \varphi(x, 0)=\chi, \quad \varphi_{t}(x, t)=\theta^{0} \\
& \varphi(x, 0)=\varphi(L, t)=0
\end{aligned}
$$


From the Theorem 2.1, we conclude that exist $\left\{\varphi^{0}, \varphi^{1}\right\}=\left\{\chi, \theta^{0}\right\} \in H_{0}^{1}(0, L) \times L^{2}(0, L)$ that satisfies the controllability of the problem (1). Consider $G$, the space defined by

$$
G=\left\{\left(-\varphi_{x}(0, t), \varphi_{x}(L, t)\right) ; \varphi(x, 0), \varphi_{t}(x, 0) \in C_{0}^{\infty}(0, L)\right\},
$$

where $\varphi(x, t)$ is the solution of the problem (11). We can define in $G$ the following quadratic form:

$$
\left\|\left\{-\varphi_{x}(0, t), \varphi_{x}(L, t)\right\}\right\|_{G}^{2}=\|\varphi(x, 0)\|_{L^{2}(0, L)}^{2}+\left\|\varphi_{t}(x, 0)\right\|_{H^{-1}(0, L)}^{2} .
$$

By the unicity of the solution $\varphi(x, t)$ of the problem (11), we conclude that $\|\cdot\|_{G}$ is a norm. Let $\bar{G}$ the completed of the $G$ with respect to the norm $\|\cdot\|_{G}$. Then $\bar{G}$ is a Hilbert space. Define by $I$ the usual isomorphism $I: \bar{G} \rightarrow \bar{G}^{\prime}$, where $\bar{G}^{\prime}$ is the dual space of $\bar{G}$. Then for $(\nu, w) \in \bar{G}$ we have

$$
I(v, w)=\left\{I_{1} v, I_{2} w\right\} \in \bar{G}^{\prime} .
$$

\section{Caracterization of the space $\bar{G}$ :}

Consider the retrograde problem

$$
\mid \begin{aligned}
& \psi_{t t}-\psi_{x x}=0 \\
& \psi(x, T)=\psi_{t}(x, T)=0 \\
& \psi(0, t)=I_{1}\left\{\theta^{0}, \theta^{1}\right\}, \quad \psi(L, t)=I_{2}\left\{\theta^{0}, \theta^{1}\right\} .
\end{aligned}
$$

and the problem (3) with the initial values

$$
\left\{\theta^{0}, \theta^{1}\right\} \in L^{2}(0, L) \times H^{-1}(0, L) .
$$

Consider the isomorphism between the spaces $L^{2}(0, L) \times H^{-1}(0, L)$ and $H_{0}^{1}(0, L) \times L^{2}(0, L)$, defined by $L:\left\{\varphi^{0}, \varphi^{1}\right\} \rightarrow\left\{\chi, \theta^{0}\right\}$.

If we apply the isomorphism on $\varphi(x, t)$, we obtain:

$$
\varphi_{x}(0, t), \quad \varphi_{x}(L, t) \in L^{2}(0, T) .
$$

On ther other hand the application

$$
D: H_{0}^{1}(0, L) \times L^{2}(0, L) \rightarrow L^{2}(0, L) \times L^{2}(0, L),
$$

defined by $D\left\{\chi, \theta^{0}\right\}=\left\{-\varphi_{x}(0, t), \varphi_{x}(L, t)\right\}$ is surjective. Taking $T>\frac{L}{2}$ we concluded that the application $D$ is a isomorphism, since

$$
\left\|\varphi_{x}(0, t), \varphi_{x}(L, t)\right\| \text { is a norm in } L^{2}(0, T) .
$$


From (7) we have that

$$
\theta_{x}(0, t)=\varphi_{x t}(0, t) \quad \text { and } \quad \theta_{x}(L, t)=\varphi_{x t}(L, t) .
$$

As the application

$$
\frac{d}{d t}: L^{2}(0, T) \rightarrow H^{-1}(0, T)
$$

is also a isomorphism, we conclude that the composition

$$
\frac{d}{d t} \circ D \circ L: L^{2}(0, L) \times H^{-1}(0, L) \rightarrow H^{-1}(0, T) \times H^{-1}(0, T),
$$

defined by $(d / d t) \circ D \circ L\left\{\theta^{0}, \theta^{1}\right\}=\left\{-\theta_{x}(0, t), \theta_{x}(L, t)\right\}$ is a bijective application. From (13) we also conclude that it is an application from $L^{2}(0, L) \times H^{-1}(0, L)$ in $\bar{G}$. This means that when $\left\{\theta^{0}, \theta^{1}\right\} \in L^{2}(0, L) \times H^{-1}(0, L)$, the normal derivate of the solution of problem (3), given by

$$
\left\{-\theta_{x}(0, t), \theta_{x}(L, t)\right\} \in \bar{G}
$$

Then,

$$
\bar{G}=H^{-1}(0, T) \times H^{-1}(0, T) \quad \text { and } \quad \bar{G}^{\prime}: H_{0}^{1}(0, T) \times H_{0}^{1}(0, T) .
$$

Consider now the retrograde problem (14). As

$$
\left\langle\Lambda\left\{\theta^{0}, \theta^{1}\right\},\left\{\theta^{0}, \theta^{1}\right\}\right\rangle=\left\|\left\{-\theta_{x}(0, t), \theta_{x}(L, t)\right\}\right\|_{\bar{G}}^{2}
$$

is a norm, then from the Lemma of Lax-Milgram, there are $\left\{\theta^{0}, \theta^{1}\right\} \in L^{2}(0, L) \times H^{-1}(0, L)$ such that the solution $\psi(x, t)$ satisfies:

$$
\psi(x, 0)=y^{0}, \quad \psi_{1}(x, 0)=y^{1}
$$

Hence we conclude the theorem.

Observation: In [5], it is proved that in the conditions of the theorem 2.2 the solution $y=y(x, t)$ has the following regularity:

$$
y \in C^{1}\left([0, T] ; L^{2}(0, L)\right) \cap C^{0}\left([0, T] ; H_{0}^{1}(0, L)\right) .
$$

\section{NUMERICAL METHOD}

Notation: Consider in the intervals $[0, L] \subset I R$ and $[0, T] \subset \mathbb{R}$, the following discretization:

$$
0=x_{0}<x_{1}<\cdots<x_{j}=L \quad \text { and } \quad 0=t^{0}<t^{1}<\cdots<t^{N}=T
$$

To all $\left(x_{j}, t^{n}\right)$ the function $y(x, t)$ will be denoted by 


$$
\begin{gathered}
y(x, t)=y\left(x_{j}, t^{n}\right)=y(j h, n k)=y_{j}^{n} \\
x_{j}=j \cdot h ; \quad j=0,1, \cdots, J \\
t^{n}=n \cdot k ; \quad n=0,1, \cdots, N .
\end{gathered}
$$

\subsection{Numerical Solution}

Consider the linear no homogeneous problem (1) where $v(t)$ and $w(t)$ are the boundary values and the Finite Difference Operator given by

$$
\delta_{h}^{2} y_{j}^{n}=y_{j-1}^{n}-2 y_{j}^{n}+y_{j+1}^{n} .
$$

A standard family of numerical methods for the problem (1) using finite difference thecnique is given by (see [9])

$$
\frac{1}{k^{2}} \delta_{t}^{2} y_{j}^{n} \approx \frac{1}{h^{2}}\left(\theta \delta_{h}^{2} y_{j}^{n-1}+(1-2 \theta) \delta_{h}^{2} y_{j}^{n}+\theta \delta_{h}^{2} y_{j}^{n+1}\right) .
$$

The numerical schemes (16) are denominated Implicit Methods for all $\theta$, $0<\theta<1$. When $\theta=0$, then we have the Explicit Method and ${ }^{*}$ if $\theta=1$, we have a Retrograde Explicit Method. If $0<\theta<1$, the numerical solution $y_{j}^{n}$ cónverge to the exact solution $y(x, t)$ with error $O\left(k^{2}+h^{2}\right)$, to all $r=\frac{k}{h}$. In particular, taking $\theta=\frac{1}{4}$, the numerical scheme is given by:

Let $n=0$,

$$
A y_{j}^{1}=\frac{1}{2} B y_{j}^{0}+k A\left(y_{t}\right)_{j}^{0}+\frac{1}{2} C^{0}, \quad j=1,2, \cdots, J-1
$$

Let $n=1,2, \cdots$,

$$
A y_{j}^{n+1}=B y_{j}^{n}-A y_{j}^{n-1}+C^{n}, \quad j=1,2, \cdots, J-1 \quad .
$$

where the column vectors $C^{n}$ and $C^{0}$ are know and given by boundary value, defined by:

$$
\begin{aligned}
C^{n} & =\frac{r^{2}}{4}\left(y_{0}^{n+1}+2 y_{0}^{n}+y_{0}^{n-1}, 0, \cdots, 0, y_{J}^{n+1}+2 y_{J}^{n}+y_{J}^{n-1}\right)= \\
& =\frac{r^{2}}{4}\left(v^{n+1}+2 v^{n}+v^{n-1}, 0, \cdots, 0, w^{n+1}+2 w^{n}+w^{n-1}\right) . \\
C^{0} & =\frac{r^{2}}{4}\left(y_{0}^{1}+2 y_{0}^{0}+y_{0}^{-1}, 0, \cdots, 0, y_{j}^{1}+2 y_{j}^{0}+y_{j}^{-1}\right)= \\
& =\frac{r^{2}}{2}\left(v^{1}+y_{0}^{0}-k\left(y_{t}\right)_{0}^{0}, 0, \cdots, 0, w^{1}+y_{J}^{0}-k\left(y_{t}\right)_{J}^{0}\right) .
\end{aligned}
$$


The matrix A and B are symmetric and tridiagonal, given by:

$$
A=\left[\begin{array}{ccccc}
a & b & & & \\
b & a & b & & \\
& & & & \\
& & b & a & b \\
& & & b & a
\end{array}\right], \quad B=\left[\begin{array}{llllll}
c & d & & & \\
d & c & d & & \\
& & & & & \\
& & d & c & d \\
& & & d & c
\end{array}\right]
$$

where

$$
a=1+\frac{1}{2} r^{2} ; \quad b=-\frac{1}{4} r^{2} ; \quad c=2-r^{2} ; \quad d=\frac{1}{2} r^{2},
$$

The numerical solution $y_{j}^{n}$, for a fixed $n$, is obtained through of the solution of the linear system.

\subsection{The Problem Description}

The goal is to determine the pair of initial data $\left\{\theta^{0}, \theta^{1}\right\} \in \underset{*}{F^{\prime}}$ such that the solution $\theta=\theta(x, t)$ of problem (3), can generate the transversal motion on boundary of the problem:

$$
\mid \begin{array}{ll}
y_{t t}-y_{x x}=0 & \text { in }] 0, L[\times] 0, T[ \\
y(x, 0)=y^{0}(x) ; \quad y_{t}(x, 0)=y^{1}(x) & \text { in }] 0, L[ \\
y(0, t)=-\theta_{x}(0, t) ; \quad y(L, t)=\theta_{x}(L, t) & \text { in }] 0, T[.
\end{array}
$$

such that at a time $T>\frac{L}{2}$, the solution $y=y(x, t)$ of problem (22) satisfies the equilibrium condicion (2), for all pair of initial data $\left\{y^{0}, y^{1}\right\}$. Notice that problem (22) is the same as problem (1), taking the boundary value

$$
\left.v(t)=-\theta_{x}(0, t) ; \quad w(t)=\theta_{x}(L, t), \quad t \in\right] 0, T[.
$$

We want to establish one relation between the functions $\left\{\theta_{x}(0, t), \theta_{x}(L, t)\right\}$ and the initial value $\left\{y^{0}, y^{1}\right\}$. Since the function $\theta_{x}$ is unique for all $\left\{\theta^{0}, \theta^{1}\right\}$ then the relation can be established between $\left\{\theta^{0}, \theta^{1}\right\}$ and $\left\{y^{0}, y^{1}\right\}$. In the HUM the relation is given by isomorphism

$$
\therefore \quad \Lambda\left\{\theta^{0}(x), \theta^{1}(x)\right\}=\left\{-y^{1}(x), y^{0}(x)\right\}
$$

We will show in the following that in the numerical case the relation is given by the linear system $S \theta=\chi$, defined bellow. 


\subsection{Application the Numerical Method}

We will first apply the numerical method (17) and (18) on problem (3), to establish the relation between the solution $\theta(x, t)$ and the initial values $\left\{\theta^{0}, \theta^{1}\right\}$. The initial values are not know and have to be determinated such that the solution $\theta(x, t)$ can generate transversal motions $\theta_{x}(x, t)$ and $\theta_{x}(L, t)$ on problem (22) and such that the solution $y(x, t)$ satisfies the equilibrium condition (2).

Consider problem (3). Due to compatibility between the initial values, we have:

$$
\theta(0,0)=\theta(L, 0)=\theta_{t}(0,0)=\theta_{t}(L, 0)=0
$$

As the boundary value is vanished for all $t$, then the column vectors $C^{n}(19)$ and $C^{0}(20)$ are also vanished. From (17), since the matrix $A$ is nonsingular, it follows that:

$$
\theta_{j}^{1}=\frac{1}{2} M \theta_{j}^{0}+K\left(\theta_{t}\right)_{j}^{0}
$$

where $M=A^{-1} B$.

Define the $(J-1) \times(J-1)$ matrix, by

$$
\begin{array}{ll}
P_{1}=I ; \quad P_{2}=\frac{1}{2} M ; \quad P_{n+1}=M P_{n}-P_{n-1}, & n=2,3, \cdots \\
Q_{1}=K \cdot I ; \quad Q_{2}=K \cdot M ; \quad Q_{n+1}=M Q_{n}-Q_{n-1}, & n=2,3, \cdots
\end{array}
$$

Then (24) can be written by

$$
\theta_{j}^{1}=P_{2} \theta_{j}^{0}+Q_{1}\left(\theta_{t}\right)_{j}^{0}
$$

Taking $n=1$ in (18), we have

$$
\theta_{j}^{2}=M \theta_{j}^{1}-\theta_{j}^{0}
$$

From (25), (26) and (27) we obtain

$$
\theta_{j}^{2}=P_{3} \theta_{j}^{0}+Q_{2}\left(\theta_{t}\right)_{j}^{0}
$$

Taking $n=2,3, \cdots$, we obtain that:

$$
\theta_{j}^{n}=P_{n+1} \theta_{j}^{0}+Q_{n}\left(\theta_{t}\right)_{j}^{0}, \quad n=1,2,3, \cdots, \quad j=1,2, \cdots, J-1 .
$$

The equality (29) establish then the relation between the solution of the problem (3) and $\left\{\dot{\theta}_{j}^{0},\left(\theta_{t}\right)_{j}^{0}\right\}$. Consider now, the application of the numerical schemes (17) and (18) to problem (22). In this case the initial values $\left\{y^{0}(x), y^{1}(x)\right\}$ are known and the boundary values are given by;

$$
\left.y(0, t)=-\theta_{x}(0, t) \quad \text { and } \quad y(L, t)=\theta_{x}(L, t), \quad t \in\right] 0, T[.
$$


Using the approximation given by finite difference methods for the function $\theta_{x}$, we obtain

$$
\begin{gathered}
-\theta_{x}(0, t)=-\left(\theta_{x}\right)_{0}^{n}=-\frac{1}{h}\left(\theta_{1}^{n}-\theta_{0}^{n}\right)=-\frac{1}{h} \theta_{1}^{n} \\
\theta_{x}(L, t)=\left(\theta_{x}\right)_{J}^{n}=\frac{1}{h}\left(\theta_{J}^{n}-\theta_{J-1}^{n}\right)=-\frac{1}{h} \theta_{J-1}^{n}
\end{gathered}
$$

This way, the boundary values are given by the approximation

$$
y_{0}^{n}=-\frac{1}{h} \theta_{1}^{n} \quad \text { and } \quad y_{J}^{n}=-\frac{1}{h} \theta_{J-1}^{n}
$$

Denoting by $L$, the i-th line of a given matrix, then from (29) we can write

$$
\begin{gathered}
\theta_{1}^{n}=L_{1}\left(P_{n+1}\right) \theta_{j}^{0}+L_{1}\left(Q_{n}\right)\left(\theta_{t}\right)_{j}^{0}, \quad j=1,2, \cdots, J-1 \\
\theta_{J-1}^{n}=L_{J-1}\left(P_{n+1}\right) \theta_{j}^{0}+L_{J-1}\left(Q_{n}\right)\left(\theta_{t}\right)_{j}^{0}, \quad j=1,2, \cdots, J-1
\end{gathered}
$$

It follows that

$$
\begin{aligned}
& y_{0}^{n}=-\frac{1}{h}\left\{L_{1}\left(P_{n+1}\right) \theta_{j}^{0}+L_{1}\left(Q_{n}\right)\left(\theta_{t}\right)_{j}^{0}\right\}, \quad j=1,2, \cdots, J-1^{\prime} \\
& y_{J}^{n}=-\frac{1}{h}\left\{L_{J-1}\left(P_{n+1}\right) \theta_{j}^{0}+L_{J-1}\left(Q_{n}\right)\left(\theta_{t}\right)_{j}^{0}\right\}, \quad j=1,2, \cdots, J-1
\end{aligned}
$$

Denoting by $v^{n}=y_{0}^{n} ; w^{n}=y_{J}^{n}$ and

$$
\hat{P}_{n+1}=\left[\begin{array}{l}
L_{1}\left(P_{n+1}\right) \\
L_{J-1}\left(P_{n+1}\right)
\end{array}\right], \quad \hat{Q}_{n}=\left[\begin{array}{c}
L_{1}\left(Q_{n}\right) \\
L_{J-1}\left(Q_{n}\right)
\end{array}\right]
$$

where $\hat{P}_{n+1}$ and $\hat{Q}_{n}$ are $2 \times(J-1)$ matrices. Substituting (32) in (30) and (31), we obtain

$$
\left[\begin{array}{c}
v^{n} \\
w^{n}
\end{array}\right]=-\frac{1}{h}\left\{\hat{P}_{n+1} \theta_{j}^{0}+\hat{Q}_{n}\left(\theta_{t}\right)_{j}^{0}\right\}, \quad j=1,2, \cdots, J-1 .
$$

Denoting by

$$
\begin{aligned}
& V^{n}=\frac{r^{2}}{4}\left(v^{n}, 0, \cdots, 0, w^{n}\right), \quad n=1,2, \cdots \\
& V^{0}=\frac{r^{2}}{4}\left(y_{0}^{1}, 0, \cdots, 0, y_{J}^{0}\right), \\
& V_{t}^{0}=\frac{r^{2}}{4}\left(-K\left(y_{t}\right)_{0}^{0}, 0, \cdots, 0,-K\left(y_{t}\right)_{J}^{0}\right) .
\end{aligned}
$$


then we can write the column vectors (19) and (20) by:

$$
\begin{aligned}
\frac{1}{2} C^{0} & =V^{1}+V^{0}+\left(V_{t}\right)^{0} \\
C^{n} & =V^{n+1}+2 V^{n}+V^{n-1} .
\end{aligned}
$$

The numerical scheme (17) and (18) can be written by:

$$
\begin{aligned}
A y_{j}^{1} & =\frac{1}{2} B y_{j}^{0}+k A\left(y_{t}\right)_{j}^{0}+\left(V^{1}+V^{0}+V_{t}^{0}\right) \\
A y_{j}^{n+1} & =B y_{j}^{n}-A y_{j}^{n-1}+\left(V^{n+1}+2 V^{n}+V^{n-1}\right) .
\end{aligned}
$$

From (36) and (37), we have:

$$
y_{j}^{n}=A^{-1}\left(R_{n} V^{1}+R_{n-1} V^{2}+\cdots+R_{2} V^{n-1}+R_{1} V^{n}\right)+\chi_{n-1} .
$$

where we define the matrix:

$$
\begin{aligned}
& R_{1}=I ; \quad R_{2}=M+2 I ; \quad R_{3}=M R_{2} ; \quad R_{n}=M R_{n-1}-R_{n-2} ; \quad n=4,5,6, \cdots \\
& \chi_{0}=\frac{1}{2} M y_{j}^{0}+K\left(y_{t}\right)_{j}^{0}+A^{-1}\left(V^{0}+V_{t}^{0}\right) \\
& \chi_{1}=M \chi_{0}-y_{j}^{0}+A^{-1}\left(V^{0}\right) \\
& \chi_{n}=M \chi_{n-1}-\chi_{n-2} ; \quad n=2,3,4, \cdots
\end{aligned}
$$

where, $M=A^{-1} B$. The unknown $V^{i}, i=1,2, \cdots, n$, must be determined in order to have $y(x, T)=y_{t}(x, T)=0$ or equivalently

$$
y_{j}^{n}=\left(y_{t}\right)_{j}^{n}=0, \text { for } n=N, N+1, \cdots .
$$

Taking $n=N$ and using the approximation given by the finite difference method, we have:

$$
\left(y_{t}\right)_{j}^{N}=\frac{y_{j}^{N+1}-y_{j}^{N}}{K}=0 \Leftrightarrow y_{j}^{N+1}=y_{j}^{N}
$$

Hence:

$$
y_{j}^{N+1}=y_{j}^{N}=0, \quad j=1,2, \cdots, J-1
$$

Therefore, as the solution is regular we can take it continuous in the boundary such that it satisfies:

$$
y_{0}^{N+1}=y_{0}^{N}=y_{J}^{N+1}=y_{J}^{N}=V^{N}=V^{N+1}=0 .
$$


Taking $n=N$ and $n=N+1$ in (38) and using (44) and (45) we obtain

$$
\begin{aligned}
& R_{N} V^{1}+R_{N-1} V^{2}+\cdots+R_{2} V^{N-1}=-A\left(\chi_{N-1}\right) \\
& R_{N+1} V^{1}+R_{N} V^{2}+\cdots+R_{3} V^{N-1}=-A\left(\chi_{N}\right)
\end{aligned}
$$

Notice: The matrices $R_{s}, s=2, \cdots, N$ of the linear system are $(J-1) \times(J-1)$ matrices. The column vectors $V^{i}, i=1,2, \cdots, N-1$ are given by:

$$
V^{i}=\frac{r^{2}}{4}\left(v^{i}, 0, \cdots, 0, w^{i}\right)
$$

where $V^{i}$ has $(J-1)$ components, with $(J-3)$ components equal to zero. The product $R_{s} V^{i}$ is a $(J-1) \times 1$ matrix. We can simplify the computation of the product using in the $(J-3)$ zero components, by means of the procedure:

Let

$$
\hat{V}^{i}=\frac{r^{2}}{4}\left(v^{i}, w^{i}\right) \text { be a } 2 \times 1 \text { column vector }
$$

and $\hat{R}_{s}$ be the $(J-1) \times 2$ matrix given by the $1^{\underline{S} t}$ and the $(J-1)^{\frac{t h}{h}}$ column of the matrix $R_{s}$.

Then the result of the product is the same, i.e.

$$
R_{s} V^{i}=\hat{R}_{s} \hat{V}_{i}
$$

For simplicity, we will consider for now on

$$
V^{i}=\hat{V}_{i}=\frac{r^{2}}{4}\left(v^{i}, w^{i}\right)
$$

and $R_{s}=\hat{R}_{s}$, the $(J-1) \times 2$ matrix.

From (33) and (48) we obtain:

$$
V^{n}=\frac{r^{2}}{4}\left[\begin{array}{c}
v^{n} \\
w^{n}
\end{array}\right]=-\frac{r^{2}}{4 h}\left(\hat{P}_{n+1} \theta_{j}^{0}+\hat{Q}_{n}\left(\theta_{t}\right)_{j}^{0}\right), \quad j=1,2, \cdots, J-1 .
$$

Consider:

$$
\begin{array}{rlrl}
F_{i} & =R_{i} \cdot \hat{P}_{(N+2-i)}, & i=2,3, \cdots, N-1, N \\
G_{i}=R_{i} \cdot \hat{Q}_{(N+1-i)}, & i=2,3, \cdots, N-1, N
\end{array}
$$




$$
\begin{array}{ll}
\hat{F}_{i}=R_{i} \cdot \hat{P}_{(N+3-i)}, & i=3,4, \cdots, N, N+1 \\
\hat{G}_{i}=R_{i} \cdot \hat{Q}_{(N+2-i)}, & i=3,4, \cdots, N, N+1 .
\end{array}
$$

Considering (49) in the linear system (46), (47), we have,

$$
\begin{aligned}
& \left(\sum_{i=2}^{N} F_{i}\right) \theta_{j}^{0}+\left(\sum_{i=2}^{N} G_{i}\right)\left(\theta_{t}\right)_{j}^{0}=\frac{4 h}{r^{2}} A\left(\chi_{N-1}\right) \\
& \left(\sum_{i=3}^{N+1} \hat{F}_{i}\right) \theta_{j}^{0}+\left(\sum_{i=3}^{N+1} \hat{G}_{i}\right)\left(\theta_{t}\right)_{j}^{0}=\frac{4 h}{r^{2}} A\left(\chi_{N}\right)
\end{aligned}
$$

Define the matrix:

$$
S=\left[\begin{array}{cc}
F & G \\
\hat{F} & \hat{G}
\end{array}\right], \quad \theta=\left[\begin{array}{c}
\theta_{j}^{0} \\
\left(\theta_{t}\right)_{j}^{0}
\end{array}\right], \quad \chi=\frac{4 h}{r^{2}}\left[\begin{array}{c}
A\left(\chi_{N-1}\right) \\
A\left(\chi_{N}\right)
\end{array}\right]
$$

Then the linear system (50), (51) is equivalent to:

$$
S \cdot \theta=\chi
$$

where

$S$ is a $2(J-1) \times 2(J-1)$ matrix, and

$$
\begin{array}{ll}
F=\sum_{i=2}^{N} F_{i}, \quad \hat{F}=\sum_{i=3}^{N+1} \hat{F}_{i} \\
G=\sum_{i=2}^{N} G_{i}, \quad \hat{G}=\sum_{i=3}^{N+1} \hat{G}_{i}
\end{array}
$$

$\theta$ is a $2(J-1) \times 1$ unknown vector

$\chi$ is a $2(J-1) \times 1$ vector of independent terms.

The linear system (52) establish then the relation between $\left\{y^{0}(x), y^{1}(x)\right\}$ that generates the matrix $\chi$ and the initial values $\left\{\theta^{0}(x) \theta^{1}(x)\right\}$. The matrix $S$ is non singular for each pair of the initial values $\left\{y^{0}(x), y^{1}(x)\right\}$, then there is a unique pair of the solution.

\section{COMPUTATIONAIS ASPECTS}

Here we take explicicity the initial values $\left\{y^{0}(x), y^{1}(x)\right\}$ and the matrix $S$ determined for some values of $r=\frac{k}{h}$. We will determinate the solution of the system and then we will obtain the boundary values and the aproximation solution.

In order to compare the results, we construct a exact control problem for which the boundary as well as the exact solution are known. We show the graphs and tables and compare the results. 


\subsection{Construction of an Exact Solution}

Consider the problem (3) and the initial values;

$$
\theta^{0}(x)=\pi \theta^{1}(x)=\frac{\sqrt{2}}{2} \cdot \operatorname{sen}(\pi x) \text { in }[0,1] \text {. }
$$

In this conditions the solution (3) is clearly given by

$$
\left.\theta(x, t)=\cos \pi\left(t-\frac{1}{4}\right) \operatorname{sen} \pi x \text { in }\right] 0,1[x] 0, T[.
$$

From (54) it follows that;

$$
\theta_{x}(0, t)=-\theta_{x}(1, t)=\pi \cos \pi\left(t-\frac{1}{4}\right) .
$$

Consider now the retrograde problem

$$
\mid \begin{array}{ll}
y_{t t}-y_{x x}=0 & \text { in }] 0, T[\times] 0,1[ \\
y(0, t)=-\theta_{x}(0, t) ; \quad y(1, t)=\theta_{x}(1, t) & \text { in }] 0, T[ \\
y(x, T)=y_{t}(x, T)=0 & \text { in }] 0,1[
\end{array}
$$

where the boundary conditions are given by (55). We want then to determine the solution $y=y(x, t)$ of problem (56). In order to have the initial and boundary values compatible, we take

$$
T=n+\frac{3}{4}, \quad n=0,1,2, \cdots
$$

Hence, we obtain from (53) and (55),

$$
\theta(x, T)=\theta_{x}(0, T)=\theta_{x}(1, T)=0, \quad \forall x \in[0,1] .
$$

Consider the function $u(x, t)$ defined by,

$$
u(x, t)=-\pi \cos \pi\left(t-\frac{1}{4}\right) \cos (2 \pi x) .
$$

Then the function $u(x, t)$ is the solution of the following retrograde problem,

$$
\mid \begin{aligned}
& u_{t t}-u_{x x}=-g(x, t) \\
& u(0, t)=-\theta_{x}(0, t) ; u(1, t)=\theta_{x}(1, t) \\
& u(x, T)=0 \\
& u_{t}(x, T)=f(x)
\end{aligned}
$$


where

$$
\begin{aligned}
& g(x, t)=3 \pi^{2} \cos \pi\left(t-\frac{1}{4}\right) \cos (2 \pi x) . \\
& f(x)=\pi^{2} \operatorname{sen} \pi\left(T-\frac{1}{4}\right) \cos (2 \pi x) .
\end{aligned}
$$

Let,

$$
y(x, t)=u(x, t)+w(x, t) .
$$

then $y(x, t)$ is the solution of the retrograde problem (56), if the function $w(x, t)$ is the solution of the following retrograde problem,

$$
\mid \begin{array}{ll}
w_{t t}-w_{x x}=g(x, t) & \text { in }] 0,1[\times] 0, T[ \\
w(0, t)=0 ; \quad w(1, t)=0 & \forall t \in] 0, T[ \\
w(x, T)=0 & \forall x \in] 0,1[ \\
w_{t}(x, T)=-f(x) & \forall x \in] 0,1[
\end{array}
$$

Fourier Series Methods: Using the Fourier Series Techniques, the solution $w(x, t)$ of problem (63) is given by:

$$
w(x, t)=C_{1}(t) \operatorname{sen}(\pi x)+\sum_{\substack{p=3 \\ \text { p:impar }}}^{\infty} C_{p}(t) \operatorname{sen}(p \pi x) .
$$

where

$$
C_{p}(t)=\mid \begin{aligned}
& -\frac{10}{3} \cos \pi\left(t-\frac{1}{4}\right)+2 \pi(T-t) \operatorname{sen} \pi\left(t-\frac{1}{4}\right) ; \text { for } p=1 \\
& \frac{4}{\left(p^{2}-1\right)} \operatorname{sen}\left(\frac{p \pi}{2}\right) \cos p \pi\left(t-\frac{1}{4}\right)+\frac{12 p}{\left(p^{2}-1\right)\left(p^{2}-4\right)} \cos \pi\left(t-\frac{1}{4}\right) \\
& \text { if } p=2 k+1 ; k=1,2, \cdots
\end{aligned}
$$

Considering (64) and (60) in (62), we obtain the solution of the retrograde problem (56), given by

$$
y(x, t)=-\pi \cos \pi\left(t-\frac{1}{4}\right) \cos (2 \pi x)+C_{1}(t) \operatorname{sen}(\pi x)+\sum_{\substack{p=3 \\ p: \text { impar }}}^{\infty} C_{p}(t) \operatorname{sen}(p \pi x) .
$$

The initial values $\left\{y^{0}(x), y^{1}(x)\right\}$ are given by

$$
\begin{aligned}
y(x, 0) & =u(x, 0)+w(x, 0)= \\
& =\frac{\sqrt{2}}{2}\left\{-\pi \cos (2 \pi x)-\frac{1}{6}(3 \pi(4 n+3)+20) \operatorname{sen} \pi x\right\}
\end{aligned}
$$




$$
\begin{aligned}
& +\sum_{\substack{p=3 \\
p: \text { impar }}}^{\infty} \frac{4}{\left(p^{2}-1\right)}\left(\operatorname{sen} \frac{p \pi}{4}+\frac{3 \sqrt{2} p}{2\left(p^{2}-4\right)}\right) \operatorname{sen} p \pi x . \\
y_{t}(x, 0) & =u_{t}(x, 0)+w_{t}(x, 0)= \\
& =\frac{\sqrt{2} \pi}{2}\left\{-\pi \cos (2 \pi x)-\frac{1}{6}(3 \pi(4 n+3)-8) \operatorname{sen} \pi x\right\} \\
& +4 \pi\left\{\sum_{\substack{p=3 \\
p: \text { impar }}}^{\infty} \frac{p}{\left(p^{2}-1\right)}\left(\cos \frac{p \pi}{4}+\frac{3 \sqrt{2}}{2\left(p^{2}-4\right)}\right) \operatorname{sen} p \pi x\right\}
\end{aligned}
$$

where we use the condition $T=n+\frac{3}{4} ; \quad n=0,1,2, \cdots$

\subsection{Numerical Results}

Consider the initial values $\left\{y^{0}(x), y^{1}(x)\right\}$ and the solution $y=y(x, t)$ of problem (56) given, respectively, in (67), (68) and (66). For the following numerical results, we have fixed the number $T=n+\frac{3}{4}=3,75$ and for the numerical resolution 'of the linear system we have used Gaussian Elimination. The matrix $S$ is obtained by a linear combination of the matrix $M=A^{-1} B$, that is nonsingular and positive defined for $0<r \leq 1$. In this case the matrix $S$ is also nonsingular and hence the numerical results are valid for $0<r \leq 1$. In the resolution of the linear system we obtain the initial values $\left\{\theta^{0}(x), \theta^{1}(x)\right\}$. Next, the approximate boundary values are obtained by

$$
\begin{aligned}
& y_{0}^{n}=-\frac{1}{h}\left\{L_{1}\left(P_{n+1}\right) \theta_{j}^{0}+L_{1}\left(Q_{n}\right)\left(\theta_{t}\right)_{j}^{0}\right\}, \quad j=1,2, \cdots, J-1 \\
& y_{J}^{n}=-\frac{1}{h}\left\{L_{J-1}\left(P_{n+1}\right) \theta_{j}^{0}+L_{J-1}\left(Q_{n}\right)\left(\theta_{t}\right)_{j}^{0}\right\} .
\end{aligned}
$$

The approximate solution $y_{j}^{n}, j=1,2, \cdots, J-1 ; n=1,2, \cdots$ is obtained through the implicit numerical method (17) and (18), using the approximate boundary values $y_{0}^{n}$ and $y_{J}^{n}$ given above. We can then compute the relative error for the boundary values and for the solution with the approximate values since the exact solution of the problem (56) is known and the exact boundary values are given by

$$
y(0, t)=y(0, t)=-\pi \cos \pi\left(t-\frac{1}{4}\right) .
$$


For the construction of the following relative error tables, we take $t=0,25+0,5 \cdot n$, $n=0,1, \cdots, 6$, we fix $r=\frac{k}{h}=\frac{1}{2}$ and take $h=\frac{1}{8}, \frac{1}{16}, \frac{1}{32}, \frac{1}{64}$. As the solution is symmetric and $y(0, t)=y(1, t)$ then it is sufficient to compute the relative error for just one side of the boundary and the approximate numerical solution $y(x, t)$ for $x \in[0 ; 0,5]$. Table 4.1 shows the relative error for the boundary value and table 4.2 shows the solution relative error for $x=0,25$.

Table 4.1

Boundary Value relative error $r=\frac{k}{h}=\frac{1}{2}$

\begin{tabular}{|c|c|c|c|c|}
\hline tlh & $\mathbf{1 / 8}$ & $\mathbf{1 / 1 6}$ & $\mathbf{1 / 3 2}$ & $\mathbf{1 / 6 4}$ \\
\hline 0,25 & $1,2 \times 10^{-2}$ & $3,2 \times 10^{-2}$ & $1,4 \times 10^{-2}$ & $6,6 \times 10^{-3}$ \\
\hline 0,75 & $1,9 \times 10^{-1}$ & $2,5 \times 10^{-2}$ & $1,9 \times 10^{-3}$ & $2,5 \times 10^{-2}$ \\
\hline 1,25 & $3,7 \times 10^{-4}$ & $1,0 \times 10^{-3}$ & $4,8 \times 10^{-3}$ & $1,0 \times 10^{-2}$ \\
\hline 1,75 & $1,5 \times 10^{-1}$ & $3,1 \times 10^{-3}$ & $9,2 \times 10^{-3}$ & $2,1 \times 10^{-2}$ \\
\hline 2,25 & $1,0 \times 10^{-3}$ & $9,4 \times 10^{-3}$ & $1,6 \times 10^{-3}$ & $8,6 \times 10^{-3}$ \\
\hline 2,75 & $4,7 \times 10^{-1}$ & $1,1 \times 10^{-1}$ & $5,6 \times 10^{-2}$ & $4,7 \times 10^{-2}$ \\
\hline 3,25 & $4,6 \times 10^{-3}$ & $1,7 \times 10^{-2}$ & $3,9 \times 10^{-3}$ & $8,8 \times 10^{-3}$ \\
\hline
\end{tabular}

Table 4.2

Relative error of the solution for $x=0,25$

\begin{tabular}{|c|c|c|c|c|}
\hline thh & $\mathbf{1 / 8}$ & $\mathbf{1 / 1 6}$ & $\mathbf{1 / 3 2}$ & $\mathbf{1 / 6 4}$ \\
\hline 0,25 & $5,1 \times 10^{-2}$ & $4,3 \times 10^{-3}$ & $1,0 \times 10^{-2}$ & $1,0 \times 10^{-2}$ \\
\hline 0,75 & $4,6 \times 10^{-3}$ & $5,4 \times 10^{-4}$ & $6,8 \times 10^{-3}$ & $2,0 \times 10^{-3}$ \\
\hline 1,25 & $5,6 \times 10^{-2}$ & $2,4 \times 10^{-3}$ & $2,2 \times 10^{-2}$ & $1,8 \times 10^{-2}$ \\
\hline 1,75 & $3,6 \times 10^{-3}$ & $7,4 \times 10^{-3}$ & $6,3 \times 10^{-3}$ & $6,3 \times 10^{-3}$ \\
\hline 2,25 & $9,6 \times 10^{-2}$ & $5,0 \times 10^{-2}$ & $2,8 \times 10^{-2}$ & $8,6 \times 10^{-3}$ \\
\hline 2,75 & $1,8 \times 10^{-2}$ & $3,3 \times 10^{-2}$ & $1,9 \times 10^{-4}$ & $1,2 \times 10^{-3}$ \\
\hline 3,25 & $8,0 \times 10^{-2}$ & $5,5 \times 10^{-2}$ & $2,3 \times 10^{-2}$ & $3,7 \times 10^{-4}$ \\
\hline
\end{tabular}


The figures 4.1, 4.2, 4.3 e 4.4 show the exact and approximate boundary values for $r=\frac{1}{2}$ and $h=\frac{1}{8}, \frac{1}{16}, \frac{1}{32}, \frac{1}{64}$ respectively, for every $t=n \cdot k ; n=0,1,2, \cdots, N$ and $N k=T=3,75$. Figure 4.5 represents the boundary value when $r=\frac{k}{h}=\frac{1}{4}$, taking $k=\frac{1}{94}$ and $h=\frac{1}{16}$.

Figure 4.6 represents the boundary value when $r=\frac{k}{h}=\frac{1}{8}$, taking $k=\frac{1}{64}$ and $h=\frac{1}{8}$.

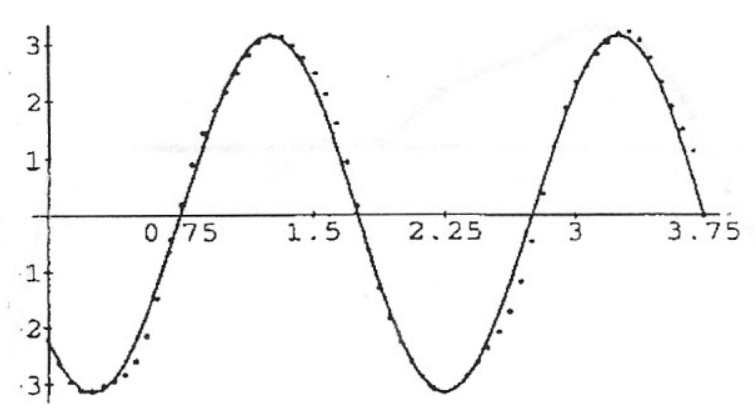

Fig. $4.1-(h, k)=(1 / 8,1 / 16)$

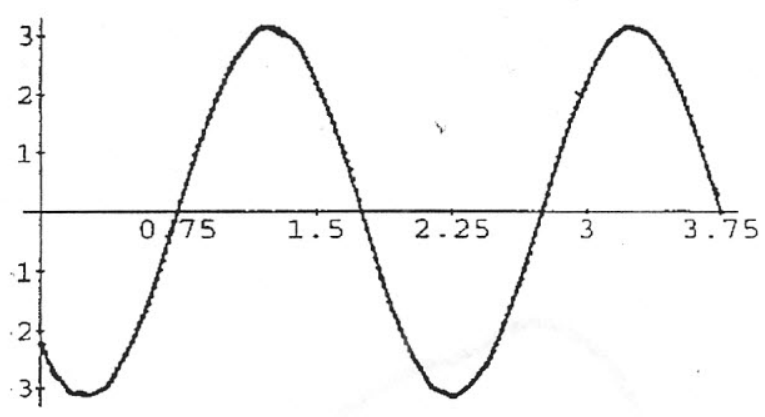

Fig. $4.3-(h, k)=(1 / 32,1 / 64)$

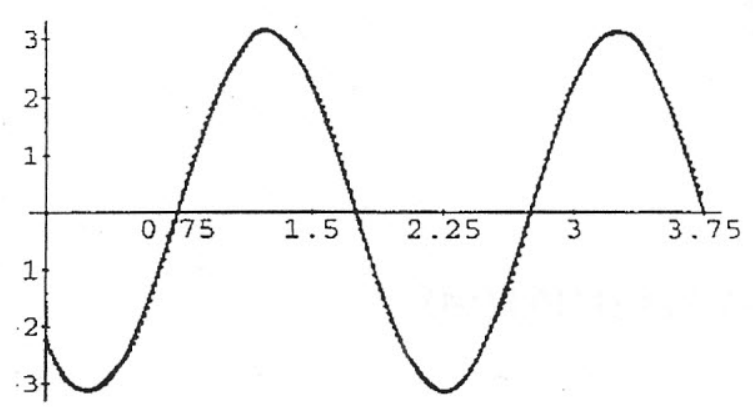

Fig. $4.5-(h, k)=(1 / 16,1 / 64)$

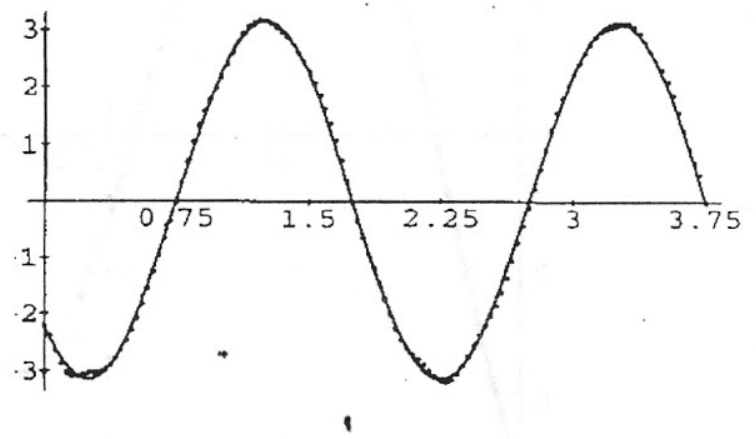

Fig. $4.2-(h, k)=(1 / 16,1 / 32)$

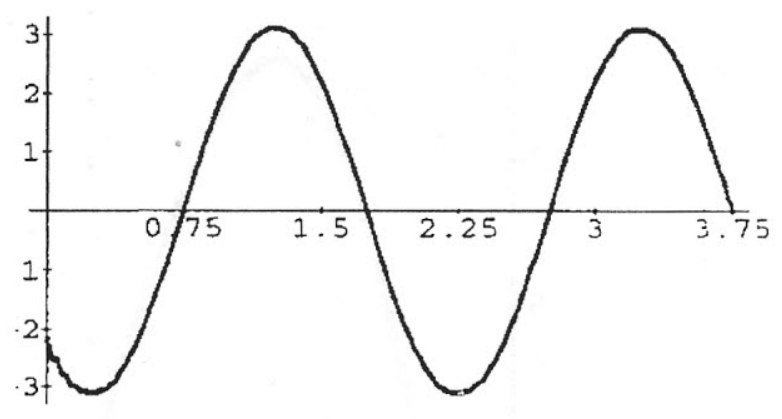

Fig. $4.4-(h, k)=(1 / 64,1 / 128)$

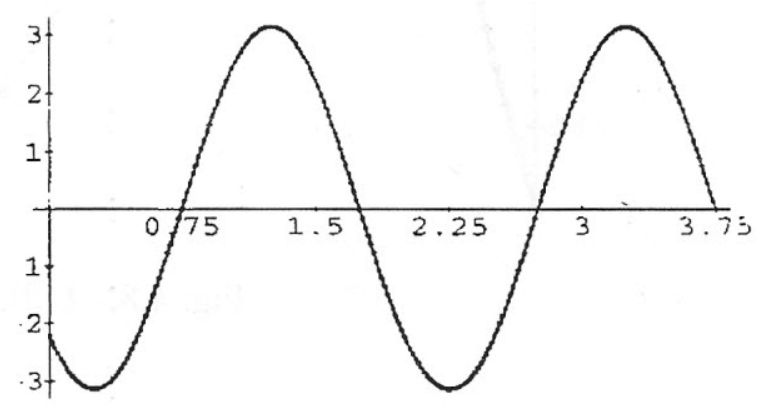

Fig. $4.6-(h, k)=(1 / 8,1 / 64)$ 
Figures 4.7 and 4.8 represent the exact and approximate solution for $x=0,25$, with $(h, k)=\left(\frac{1}{16} ; \frac{1}{32}\right)$ e $(h, k)=\left(\frac{1}{16} ; \frac{1}{64}\right)$ respectively.

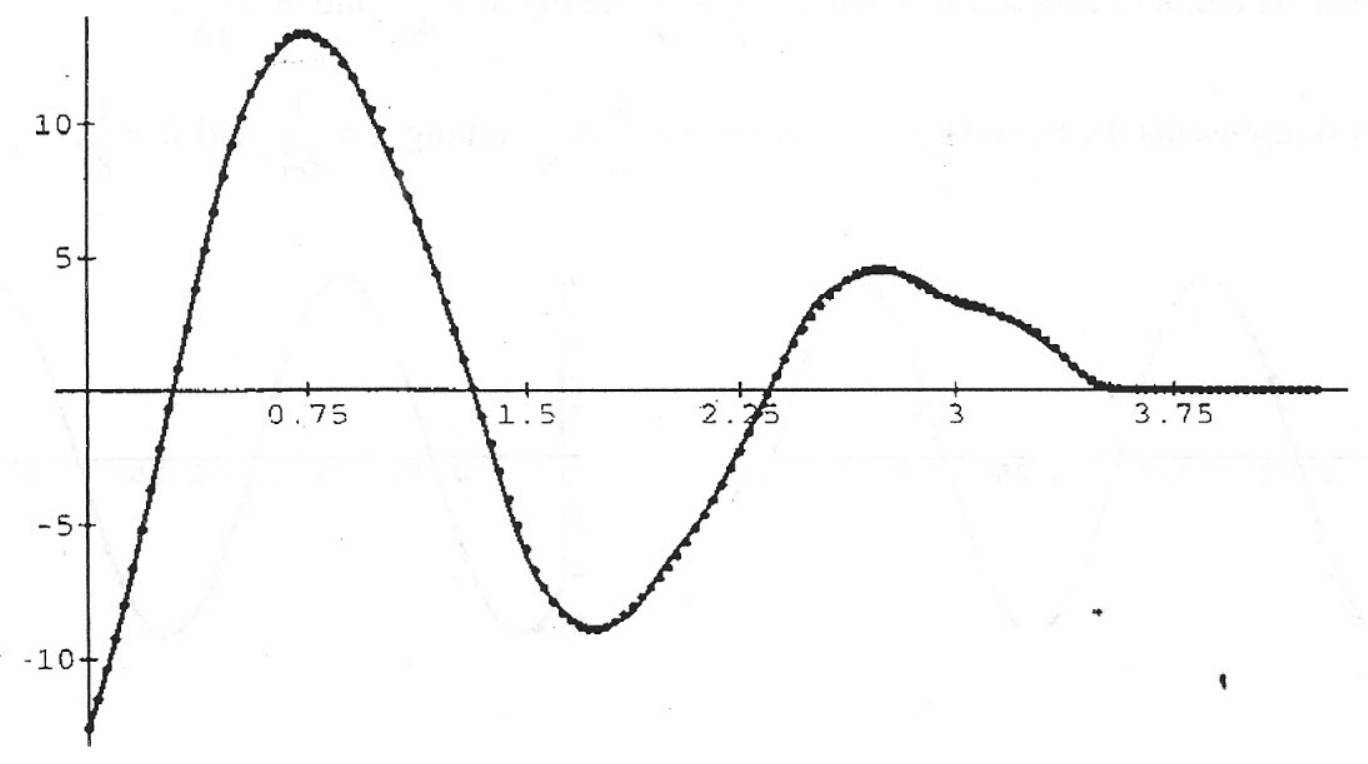

Fig. $4.7-U(0.25 ; t)-(h, k)=(1 / 16,1 / 32)$

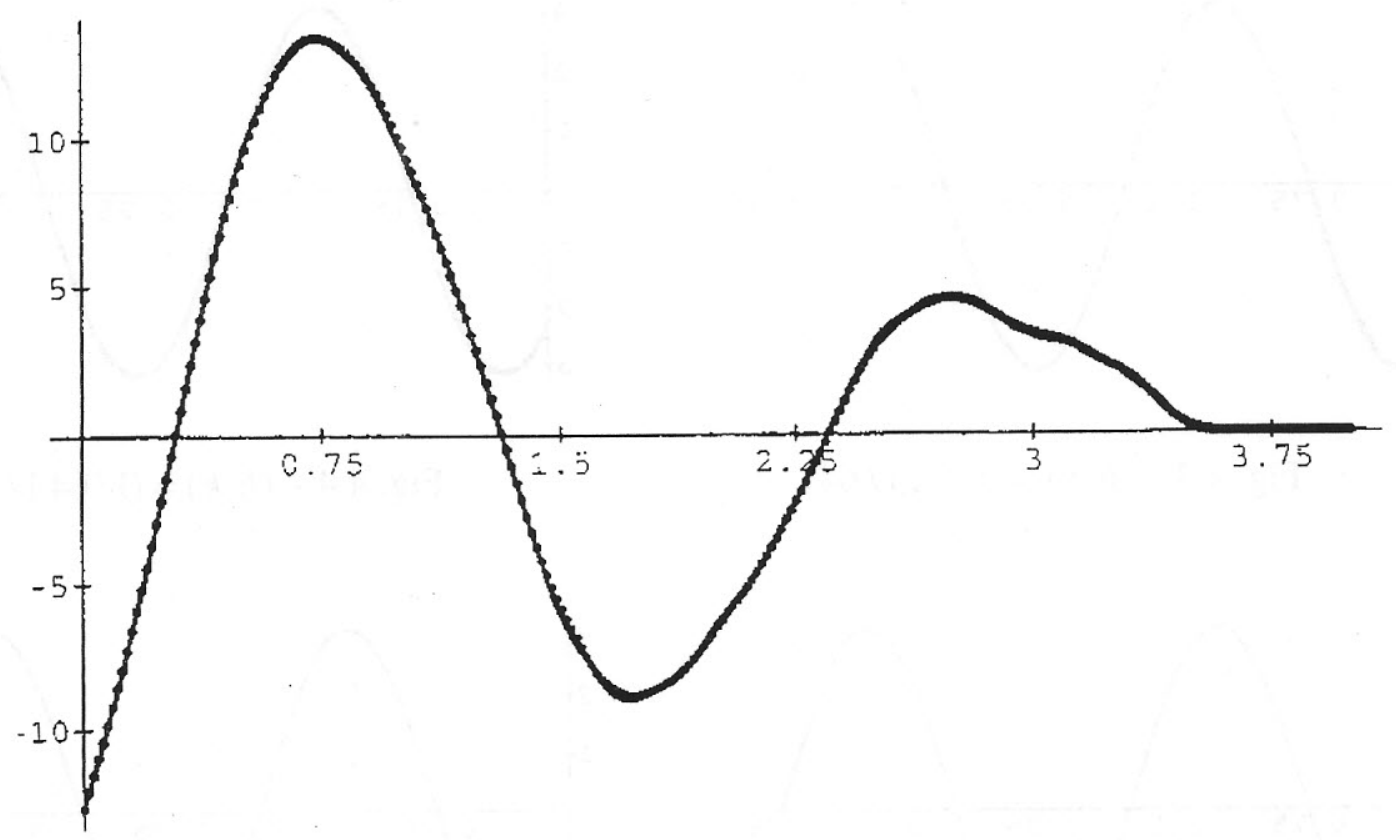

Fig. $4.8-U(0.25 ; t)-(h, k)=(1 / 16,1 / 64)$ 


\section{REFERENCES}

1. BRÉZIS, H., Análisis Funcional, Teoria y Aplicaciones, Masson, Paris (1983)..

2. LIONS J. L., Contrôlabilité Exacte, Perturbations et Estabilization de Systèmes Distribués, Tome 1, Contrôlabilité Exacte, Masson, RMAS, Paris (1988).

3. GLOWINSKI, R.; CHIN-HSIEN LI \& LIONS J.L., A Numerical Approach to the Exact Boundary Controllability of the Wave Equation(I) Dirichlet Controls: Description of the Numerical Methods, Japan Journal of Applied Mathematics, Vol. 7, N 1, pp. 1-76, (1990).

4. LiONS, J. L. \& MAGENES, E., Problèmes aux Limites Non Homogènes et Applications, Vol. 2, Dunod, Paris (1968).

5. LASIECKA, I. \& LIONS, J. L. \& TRIAGGIANI, R., J. Math Pures et Appl., 65, pp. 149-192, (1986).

6. MEDEIROS, L. A., Exact controllability for wave equations, HUM, $37^{\circ}$ Seminário Brasileiro de Análise, (1993).

7. MUÑOZ RIVERA, J., Tópicos em E.D.P: e Controle Ótimo: Controle Exato, Seminário Brasileiro de Análise, (1991).

8. RINCON, M. A., A numerical Method for the Exact Control, Estudos e Comunicações do IM/UFRJ, $\mathrm{N}^{\circ} 81$ - Rio de Janeiro - Brasil, (1996).

9. SMITH, G. D., Numerical Solution of Partial Differential Equations. Finite Difference Methods, Clarendon Press, (1978).

10. ZUAZUA, E., Controlabilidad Exacta y Estabilización de la Ecuación de Ondas, Vol. 23, IM/UFRJ (1994). 